\title{
Urban population structure and local movement ecology of a native Australian mosquito involved in disease transmission
}

\author{
Véronique Paris, Rahul Rane, Peter Mee, Stacey Lynch, Ary A Hoffmann, Thomas L Schmidt
}

VP - orcid.org/0000-0002-3667-685X

$A A H$ - orcid.org/0000-0001-9497-7645

TLS- orcid.org/0000-0003-4695-075X

\begin{abstract}
Successful species management is reliant upon understanding key ecological features of the target species and the environment. Dispersal is a critical factor to assess when designing control measures as it determines the rate of migration out of or into controlled areas and influences the risk of human exposure to the species and its pathogens. This study uses spatial population genomics to investigate the movement ecology of Aedes notoscriptus, an important disease transmitting mosquito, at the Mornington Peninsula, Australia. We sampled Ae. notoscriptus eggs from the Mornington Peninsula at a single time point, and generated genomic data from 240 individuals from different locations. We also produced a draft genome assembly for this clade of Ae. notoscriptus. We used genomic data to detect close kin dyads, and the locations of dyads showed that specific acts of movement in the previous generation had occurred over distances $>1 \mathrm{~km}$. We also investigated isolation by distance patterns from the spatial autocorrelation of genetic distances. Significant genetic dissimilarity in Ae. notoscriptus began to be observed at $>4 \mathrm{~km}$ separation, a fourfold higher distance than in a comparable sample of the dengue mosquito Ae. aegypti. These findings are evidence that the high mobility of Ae. notoscriptus influenced the success of a 2021 mosquito control trial at the Mornington Peninsula, because the dispersal ability of Ae. notoscriptus was likely to have exceeded the size of the intervention zones which were designed around ecological knowledge of Ae. aegypti. Further sampling within the same area was used to obtain counts of Ae. notoscriptus eggs at two timepoints 6 and 12 months after initial sampling. We found egg counts to be consistent across timepoints, and spatial variation in egg counts was found to covary with spatial variation in neighbourhood size (NS). As NS increases linearly with population density, this is evidence that egg counts may be useful for estimating relative density in Ae. notoscriptus. The overall results of this study draw attention to the importance of acquiring species-specific data when planning control measures, and contribute to the fundamental ecological understanding of this important vector species.
\end{abstract}




\section{Introduction}

The impact of climate and global change continue to have serious consequences for the diversity and abundance of most organisms, highlighting the importance of increased protection of threatened species as well as the control of pests. The development of sustainable and successful species management strategies in conservation, agriculture and in disease vector control relies on a fundamental understanding of key ecological features of the target species and its environment. Pest insects can have significant consequences for biosecurity by crop destruction and the spread of diseases in plants, animals, and humans. Mosquitoes are particularly important vectors, transmitting some of the most significant infectious diseases to humans, such as malaria (Beier 2003) and dengue (Bhatt et al. 2013). Populations of mosquitoes can be controlled in several ways, ranging from the use of insecticides (McCarroll et al. 2000) or traps (Juarez et al. 2021) to reduce populations sizes to the use of endosymbionts such as Wolbachia to supress or manipulate populations (Hoffmann et al. 2011).

Dispersal is a key ecological characteristics, and understanding dispersal can be vital for successful species management. Dispersal can influence human exposure to pathogen transmission as well as migration from or into controlled areas, and is therefore critical data to acquire when planning management strategies. Intrinsic and extrinsic factors such as dispersal barriers (natural or anthropogenetic) (Goldberg and Lande 2015, Schmidt et al. 2018), urbanisation (Johnson and Munshi-South 2017) and habitat fragmentation (Doak et al. 1992) can all influence dispersal and are often unique to the specific target location. The incorporation of results from dispersal studies on fine and broad scales has already positively impacted the design of pest control strategies. For example, research of the larval dispersal of the fruit orchard pest Operophtera brumata in Norway, led to the development of forecasts as to when control measures need to be implemented to mitigate the damage of plants while decreasing the use of insecticides (Edland 1983, Jeger 1999). Knowledge of the movement ecology of the malaria vector Anopheles gambiae has helped improve strategies to decrease the risk of disease transmission to humans (Killeen et al. 2003, Thomas et al. 2013, Saddler et al. 2019). 
Numerous methods have been deployed to parameterise and observe dispersal which differ in their applicability for mosquitoes. Mark-Release-Recapture (MRR) had been successfully and sufficiently used to assess individual movement of large animals, though can come with drawbacks if applied to small organisms like mosquitoes as they can be labour intensive and re-capture rates can be insufficient. An expansion of MRR approaches incorporates genetic inferences of close kin, functioning on the basis that an individual's genotype can be considered a "recapture" of the genotypes of each of its parents. This close kin mark recapture (CKMR) framework has mainly been used to investigate abundance in big populations as well as to assess migration between populations (Bravington et al. 2016). Recent studies expanded CKMR approaches, using genome wide sequence data to detect dispersal by assigning dyads to kinship categories across multiple orders of kinship and then used the spatial distribution of these kin to reveal past movement over fine temporal scales (Schmidt et al. 2018, Combs et al. 2018, Fountain et al. 2018, Jasper et al. 2019, Trense et al. 2021, Schmidt et al. 2021). Dispersal inferences from close kin treat dispersal as a set of discrete events reflecting specific acts of past individual movement, making them particularly useful for investigating dispersal through regions of genetic similarity, such as where a population has been sampled continuously across a range. These recent studies revealed the power of genetic approaches to estimate dispersal in small organisms and demonstrated an opportunity to use the genetic data acquired for additional analyses that go beyond dispersal, such as investigations of population structure and dynamics (e.g., neighbourhood size; level of gene flow).

Here we use spatial population genomics to investigate the population structure and finescale movement of Aedes notoscriptus (Australian backyard mosquito), a container breeding mosquito, native to mainland Australia and Tasmania and with invasive populations in the Torres Strait islands, New Zealand, New Guinea, New Caledonia, Indonesia (Dobrotworsky 1965, Lee et al. 1987, Sunahara and Mogi 2004) as well as to California, USA (Metzger et al. 2021). This species is known to be a vector for arboviruses (e.g. Ross-River-Virus \& BarmahForest-Virus) and is also the primary vector of dog heartworms (Russell and Geary 1992, Doggett and Russell 1997, T.M. and B.H. 1999). In the study area at the Mornington Peninsula, Victoria, this species is the main suspect of transmitting Buruli ulcer (BU) to humans. Even though this mosquito is an important vector species, threatening human and 
animal health, little research focuses on its population dynamics in the field, which makes risk calculations and the planning of interventions difficult.

Studies investigating dispersal and population structure in Aedes mosquitoes have mainly focused on Aedes aegypti (e.g. Muir and Kay 1998, Schmidt et al. 2018, Jasper et al. 2019) and the species' limited dispersal has been used as a proxy for the movement of related container breeding mosquito species (Watson et al. 2000b). However, the dispersal behaviour of different mosquito species can vary greatly due to different flight abilities, feeding and breeding preferences, and likelihood of being passively moved (Verdonschot and Besse-Lototskaya 2014), stressing that generalisations even between related species can potentially be problematic. Past MRR studies, comparing dispersal in adult Ae. aegypti and Ae. notoscriptus in Queensland present contradicting results with Watson et al. (2000) describing similar limited dispersal in both species, while Trewin et al. (2019) conclude that Ae. notoscriptus disperses further than Ae. aegypti and seems to be less restricted by barriers such as roads. In this study, we compare patterns of spatial genetic structure between Ae. notoscriptus collected in Victoria and Ae. aegypti collected in Cairns, Queensland in 2014. By investigating how these related container breeding mosquitoes vary in their genetic structure over fine scales, we will contribute to our understanding about how comparable dispersal of container breeding Aedes mosquitoes can be.

Additionally, we will discuss whether the dispersal abilities of Ae. notoscriptus estimated in this paper, affected the outcome of a pilot mosquito intervention study at the Mornington Peninsula in early 2021. Efforts were made to non-chemically reduce numbers of $A e$. notoscriptus in a randomized control trial to further investigate the role of this mosquito species in the transmission of Buruli ulcer. The study employed gravid traps over a period of four weeks to remove female mosquitoes and their offspring from the environment, resulting in no measurable reduction in numbers of $A e$. notoscriptus. Several factors could have influenced the outcome of the intervention, including the limited knowledge about $A e$. notoscriptus' movement abilities in urban environments at the time of planning. In addition, the success of the trial was evaluated using egg count data, assessed before and after control measures were in place to estimate whether the intervention had decreased the number of mosquitoes (and therefore the number of eggs in traps). We compare egg count 
data and neighbourhood size estimates throughout the study area to discuss whether egg counts sufficiently reflect mosquito numbers of Ae. notoscriptus.

\section{Material \& Methods}

\subsection{Fine scale population structure and movement}

\subsubsection{Sampling of Ae. notoscriptus}

Aedes notoscriptus were collected in February 2019 from four locations at the Mornington Peninsula: Sorrento (north-west), Blairgowrie (central) and Rye (north-east and south-east). Collections deployed an oviposition trap consisted of a black plastic bucket, halfway filled with water containing several alfalfa pellets to attract gravid Ae. notoscriptus (Ritchie 2001). A strip of red felt extending into the water provided an oviposition substrate. Felts were collected after 7 and 14 days and partially dried. Three days after collection, eggs were hatched in $500 \mathrm{~mL}$ reverse osmosis (RO) water containing 2-3 TetraMin tropical fish food tablets (Tetra, Melle, Germany). If no larvae hatched, felts were re-dried for three days and the hatching process repeated. Water and food were replaced as appropriate. Emerging virgin adults were transferred into absolute ethanol and stored at $-20^{\circ} \mathrm{C}$ until DNA extraction. One individual mosquito per trap was randomly chosen for DNA sequencing, resulting in 240 individuals being processed.

\subsubsection{DNA extraction and library preparation}

Mosquitoes were morphologically identified using keys from Webb et al. (2016) and DNA was extracted from individual mosquitoes using either Qiagen DNeasy Blood \& Tissue Kits (Qiagen, Hilden, Germany) or Roche High Pure ${ }^{\mathrm{TM}}$ PCR Template Preparation Kits (Roche Molecular Systems, Inc., Pleasanton, CA, USA) following the manufacturer's instructions. We prepared double-digest restriction site-associated DNA sequencing (ddRADseq) libraries starting with an initial digestion of $30-200 \mathrm{ng}$ of genomic DNA, using 10 units each of $\mathrm{MluCl}$ and Nlalll restriction enzymes, NEB CutSmart buffer (New England Biolabs, Beverly MA, USA), and water. Digestions were run for 3 hours at $37^{\circ} \mathrm{C}$ with no heat kill step, and the products were cleaned with paramagnetic beads. Modified Illumina P1 and P2 adapters were ligated onto cleaned digestions overnight at $16^{\circ} \mathrm{C}$ with 1,000 units of T4 ligase (New 
England Biolabs, Beverly, MA, USA), followed by a 10-minute heat-deactivation step at $65^{\circ} \mathrm{C}$. Size selection was performed using a Pippin-Prep 2\% gel cassette (Sage Sciences, Beverly, MA) to retain DNA fragments of $350-450 \mathrm{bp}$.

The size selected libraries were amplified by PCR, using $1 \mu \mathrm{L}$ of size-selected DNA, $5 \mu \mathrm{L}$ of Phusion High Fidelity 2x Master mix (New England Biolabs, Beverly MA, USA) and $2 \mu \mathrm{L}$ of 10 $\mu \mathrm{M}$ standard Illumina P1 and P2 primers. These were run for 12 PCR cycles, then cleaned and concentrated using $0.8 x$ paramagnetic beads. Each ddRAD library contained 24 mosquitoes, and each was sequenced on a single sequencing lane using 150 bp chemistry. Libraries were sequenced paired end at GeneWiz, Inc (Suzhou, China) HiSeq 4000 (Illumina, California, USA).

\subsubsection{Data processing}

We used the Process_radtags program in Stacks v2.0 (Catchen et al. 2013) demultiplex sequence reads. Using a 15 bp sliding window, low quality reads were discarded if the average phred score dropped below 20. We used Bowtie v2.0 (Langmead and Salzberg 2012) to align reads to the Ae. notoscriptus reference genome assembly (described in 2.1), using -very-sensitive alignment settings. All alignments were filtered to paired reads that aligned concordantly, requiring the two paired reads to align to the same contig to avoid multimapping using Samtools (Danecek et al. 2021). Stacks Ref_map program was used to build Stacks catalogs, from which genotypes were called at RAD stacks at a 0.05 significance level and --min-mapq 15 to filter any remaining multi-mapped reads. We generated VCF files for the catalog with the Stacks program Populations (Catchen et al. 2013). SNPs were required to be scored in $\geq 90 \%$ of mosquitoes, with a minor allele count of $>3(-r 0.90-$ mac $3--v c f)$. Beagle v4.1 (Browning and Browning 2016) was used to impute and phase the dataset in a 50,000 bp sliding window and with 3,000 bp overlap. Finally, vcftools was used to thin SNPs so that no two SNPs are in 500 bp distance to each other (--thin 500). After filtering we retained 11,091 SNPs.

\subsubsection{Genetic diversity and local population structure}

Populations was used to calculate pairwise $\mathrm{F}_{\mathrm{ST}}$ between all samples as well as between the four sampling sites. Isolation By Distance (IBD) between all samples as well as within each of the four sampling sites was tested using the mantel.randtest function in the R package 
'ade4' (Dray and Dufour 2007). We also tested for IBD of the dataset after removing pairs of individuals that have been identified as close kin to further investigate the influence of fine scale dispersal on local genetic structure. The simple Mantel test analysed matrices of pairwise genetic distance and the natural logarithm of Haversine pairwise geographic distance, employing 9,999 permutations and Bonferroni correction to assess statistical significance. Rousset's a (Rousset 2000) provided genetic distances, calculated in SPAGeDI (Hardy and Vekemans 2002). Additionally, we used the pairwise genetic distance and geographical distance matrices to measure spatial autocorrelation using the mgram function in the R package "ecodist" (Goslee and Urban 2007) to build correlograms.

To contextualise these spatial genetic structure results, we compared results for $A e$. notoscriptus with an Ae. aegypti population from Cairns, Australia, sampled in 2014 using similar protocols and with similar spatial distributions of traps (Schmidt et al. 2018). This Ae. aegypti dataset contained both, individuals carrying a Wolbachia infection (wMel) from recent releases in the area and wildtype individuals without the infection (WT), and we analysed these separately to avoid bias. We downsampled each Ae. aegypti dataset to only include one sample per trap to achieve maximum comparability to the Ae. notoscriptus dataset.

Neighbourhood size (NS: Wright 1946) was estimated using the inverse of the regression slope of pairwise individual genetic distance (Rousset's $a$ ) against the natural logarithm of geographical distance (Rousset 2000). NS represents the effective number of $A e$. notoscriptus that contribute to the local breeding 'neighbourhood' when isolation by distance is operating. We used the $R$ package 'sGD' (Shirk and Cushman 2011) to estimate spatially explicit indices of Wright's neighbourhood size (NS) in continuous populations isolated by distance (Shirk and Cushman 2014). The genetic neighbourhood radius was determined by the distance class that showed the most significant positive genetic correlation calculated in the mgram function described above (i.e., $1300 \mathrm{~m}$ ). We set the minimum population size to 20 individuals to minimize sampling error. We used the defined local neighbourhood around each sampling location to interpolate NS throughout the entire study area. Ordinary Kriging was performed in $R$ using the ' $g e o R^{\prime}$ package to interpolate data on a map and visualize the pattern of NS across the sampling area. We fitted several 
semivariogramms with different covariance models and the model returning the lowest SSQ value was chosen as the best fitting model for the data. Results returned by the Kriging model were cross validated using the xvalid function which performs model validation by comparing observed values and values predicted by kriging. Visualization of results was achieved though the image function.

To further investigate the local population structure of Ae. notoscriptus and possible coancestry between individuals of the different sampling sites, we used the program fineRADstructure (Malinsky et al. 2018) which was run with default settings.

\subsubsection{Local movement estimates of individuals}

We investigated the association between kinship and distances between all samples to infer specific movements of the parental generation, treating separation distances between pairs of kin as representatives of past dispersal events. The distances between full-siblings result from the mother's oviposition dispersal and therefore represent the direct movement of a single individual female between two traps. Finding a mate of the father as well as the host seeking and oviposition dispersal of each individual mother result in distances of halfsiblings as female Aedes mosquitoes usually mate once (Christophers, 1960), while males mate with several females. First cousins are separated through the ovipositional dispersal of their grandmother in addition to the premating dispersal of each parent, plus the postmating dispersal and ovipositional dispersal of each mother. We generated kinship coefficients using PC-Relate (Conomos et al. 2016), which conditions the data with principal components (PCs) to control for genetic structure. We generated kinship coefficients for all dyads following different conditioning treatments, ranging from 2PCs up to 30 PCs.

Reviewing the PC plots revealed that 4PCs conditioned the data the best, as it showed tight clustering of individuals. Kinship classes were defined as full-siblings kin $\geq 0.1875$, half siblings $\geq 0.09375$ and first cousins $\leq 0.07$.

\subsubsection{Analyses of egg count data}

Egg count data for all four sampling sites was acquired in additional sampling in November 2019 and February 2020, using oviposition traps as described in 2.1. All eggs found on 120 
felts per timepoint were counted by hand by a single person to ensure consistency. The data was used to investigate whether egg counts differed between sites and if they were consistent over both time points. Additionally, we compared predicted egg counts throughout the sampling area with predicted NS, calculated in 'sGD' (see 2.2.4) to discuss whether egg counts can be used as a predictor for NS.

\subsubsection{Graphical analyses}

Ordinary Kriging was performed in $R$ using the "geoR" package to interpolate data on a map and visualize the pattern of egg numbers across different sampling areas. We created interpolative maps predicting egg numbers throughout the study area for both timepoints separately to investigate if trends stay consistent throughout the mosquito season. Egg numbers were cube transformed to archive normal distribution before semivariogramms with different covariance models were fitted. The model returning the lowest SSQ value was chosen as the best fitting model for the data. Results returned by the Kriging model were cross validated using the xvalid function which performs model validation by comparing observed values and values predicted by kriging. Visualization of results was achieved though the image function. Eggs counts were back transformed before being plotted onto the map.

\section{Results}

\subsection{Population structure and movement}

\subsubsection{Genetic diversity and local population structure}

Pairwise $F_{\text {st }}$ estimated between zones can be found in Table 1.

Table 1: $F_{\text {st }}$ estimates between sampling zones

\begin{tabular}{|l|l|l|l|l|}
\hline & North-west & Central & South-east & North-east \\
\hline North-west & & 0.00163 & 0.00165 & 0.0013 \\
\hline Central & & & 0.0008 & 0.0008 \\
\hline North-east & & & & \\
\hline
\end{tabular}


The fineRADstructure plot (Figure 1) shows that there is no clear clustering of coancestry between mosquito pairs collected from the same sampling sites which indicates gene flow between all sites.

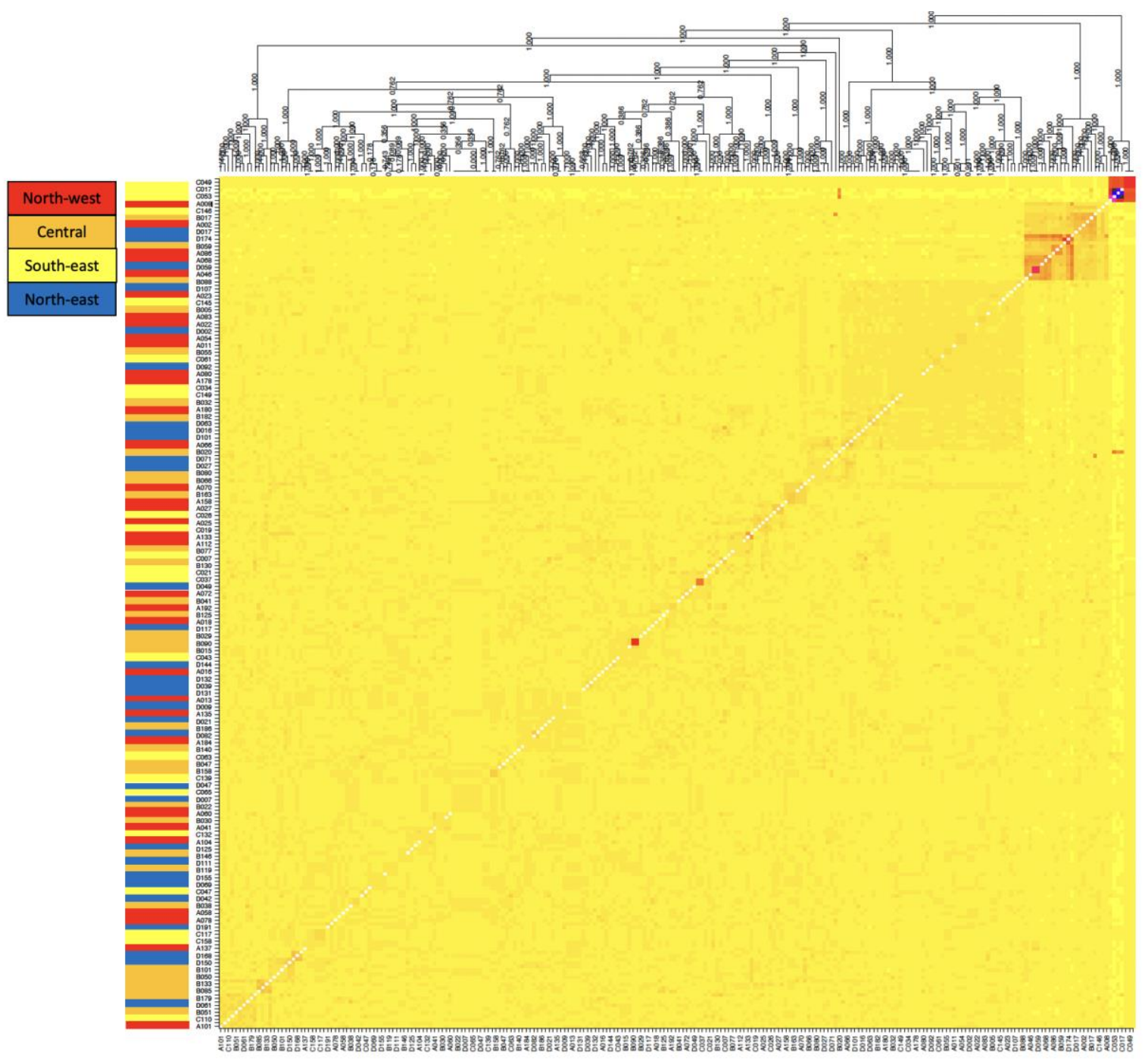

Figure 1: FineRadstruture coancestry map and tree. The left-hand side panel indicates genotype sampling site ('North-west' in red; 'Central' in orange; 'South-east' in yellow; 'North-east' in blue).

Moderate isolation by distance was revealed by the positive relationship between genetic distance and the natural logarithm of geographical distance (Bonferroni-corrected P-value $<0.05, r=0.05$ ) if calculated on the entire Ae. notoscriptus dataset (mean distance between trap pairs $=2045 \mathrm{~m}$ ). If related individuals were removed from the dataset, no IBD could be detected, indicating that IBD at the scale of the whole sampling area is driven by the local 
dispersal of related individuals, which is consistent with pattern described in Aguillon et al. (2017). No pattern of IBD could be detected if tested within each sampling site, where the mean distances of trap pairs was $522 \mathrm{~m}$, suggesting little genetic structure of Ae. notoscriptus at this scale. In contrast, Ae. aegypti populations showed IBD at a similar geographical scale (mean trap pair distance: WT: 739m; wMel: 613m) (Bonferroni-corrected P-value $<0.001)$ indicating local genetic structure in Ae. aegypti populations.

Spatial autocorrelation was positive and significant among Ae. notoscriptus samples around the range of $1300 \mathrm{~m}(r=0.02, p=0.005)$ as well as at $3700 \mathrm{~m}(r=0.03, p=0.02)$. At distances ranging from $4700 \mathrm{~m}$ onwards, Ae. notoscriptus showed significantly negative autocorrelation $(r=-0.05, p<0.001)$ where individual mosquitoes were effectively no more related than they would be at random. The mean distance between traps was $1045.95 \mathrm{~m}$ (Figure 2A). Aedes aegypti signature of spatial structure showed positive and significant values in the first distance class of $100 \mathrm{~m}$ (WT: $r=0.08, p=0.02$; wMel: $r=0.05, p=0.001$ ), then decreased sharply, dropping below zero beyond 500m. Significant negatively autocorrelated values are estimated from around 1100m (WT: $r=-0.104, p=0.005$; wMel: $r=-0.05, p=0.01$ ). The mean trap distance for WT Ae. aegypti was $739.14 \mathrm{~m}$ and $613.62 \mathrm{~m}$ for the wMel infected population (Figure $2 \mathrm{~B}+\mathrm{C}$ ). 

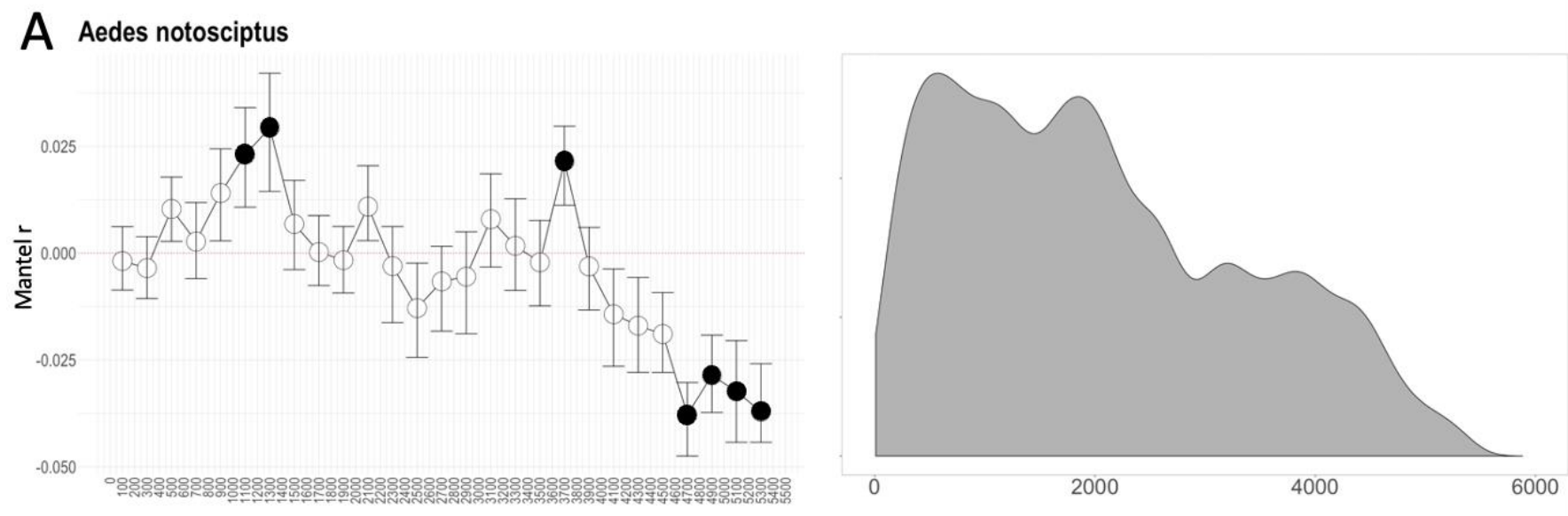

B Aedes aegypti-wildtype
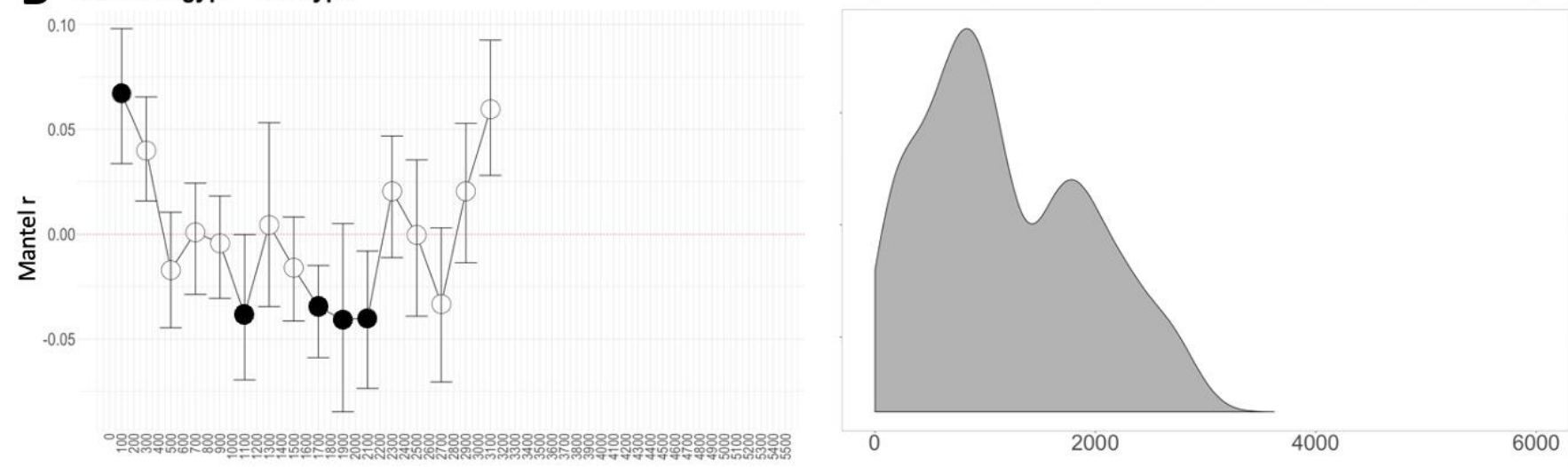

\section{Aedes aegypti-wMel infected}
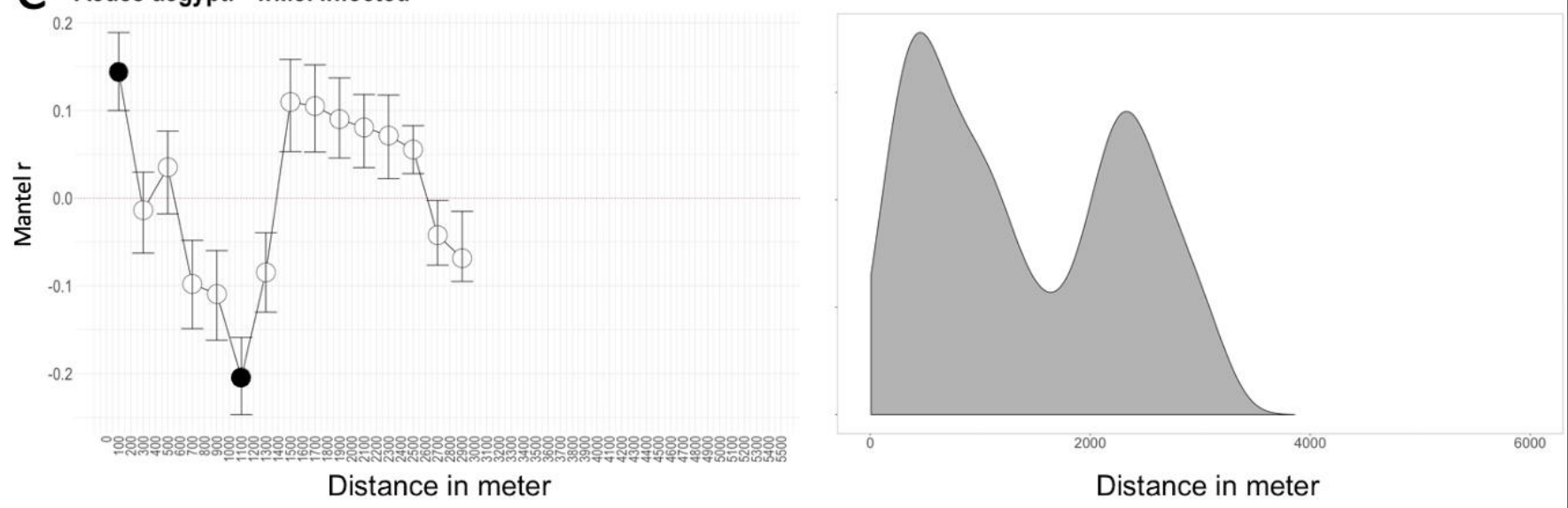

Figure 2: Spatial autocorrelation (left) and density of trap distances (right) of Aedes notoscriptus (A), WT Aedes aegypti (B) and wMel infected Aedes aegypti (C). Black filled circles indicate significant values. 
bioRxiv preprint doi: https://doi.org/10.1101/2022 01.18.476837; this version posted January 21, 2022. The copyright holder for this preprint (which was not certified by peer review) is the author/funder, who has granted bioRxiv a license to display the preprint in perpetuity. It is made available under aCC-BY-NC 4.0 International license.

\subsubsection{Local movement estimates of individuals}

We identified three putative full-sibling and 8 half-sibling pairs using PC-relate. We also designated 8 pairs with $k>0.07$ as putative first cousins. First order relatives were separated by a mean distance of $466 \mathrm{~m}$ (median=179m) and exhibited a maximum observed distance of $1267 \mathrm{~m}$. The mean separation distance for second order relatives was $1296 \mathrm{~m}$. (median=340m, $\max =5173 \mathrm{~m}$ ); and third order relatives' mean distance was $2778 \mathrm{~m}$ ( median=2825m, $\max =4664 \mathrm{~m}$ ) (Figure 3A).

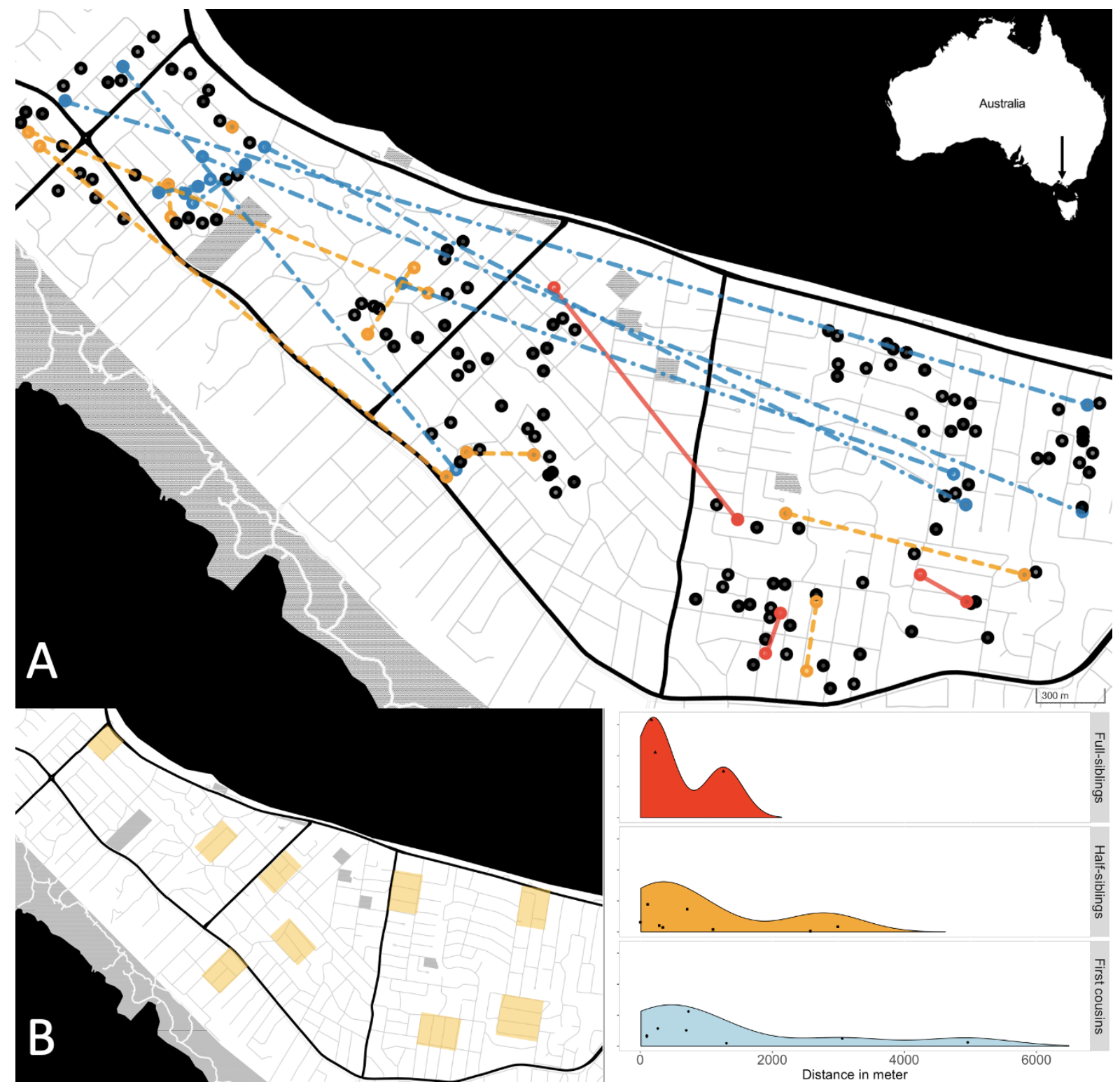

Figure 3: Trap placement, kinship network and distribution and intervention zones at the Mornington

Peninsula. (A) Circles represent traps of which one individual mosquito was chosen for sequencing. Lines indicate pairs of full-siblings (red, solid), half-siblings (orange, single-dashed) and first cousins (blue, doubledashed). (B left) Zones of the 2021 mosquito intervention pilot study are represented as yellow rectangles. (B right) Distribution of trap distances in meters of full-siblings (red), half-siblings (orange) and first cousins (blue). 
Two of the full-sibling pairs were found within the same site (south-east), while the third full-sibling pair was found between the central and south-east sites (Figure 2A). Most of the half-sibling pairs were detected within the same site (north-west, central, and south-east), with one pair found in the same trap one week apart and two pairs were distributed between the north-west and central sites (Figure 3A). Pairs of first cousins moved between the north-west site and the sites in the north and south-east with just three of the pairs found within the same site (north-west) (Figure 3A).

\subsection{Eggs counts and neighbourhood size (NS)}

\subsubsection{Graphical analysis of egg counts}

Predicted egg counts throughout the entire sampling area were estimated by ordinary kriging for each time-point and are presented in Figure 4. Predictions were based on cube transformed egg count data to achieve normal distribution, however egg counts per trap location were plotted after back transformation to represent real egg counts per trap. The plots show a pattern of increased egg counts from east to the north-west sites. The estimated prediction error after cross validation indicates that on an average, an error of 1.498 of mean egg counts can be expected at any given location. Patterns of spatial variation in egg counts were consistent across the two time points (Nov 2019 and Feb 2020).

\subsubsection{Graphical analysis of neighbourhood size (NS)}

The calculation of NS for each sampling location completed using ' $s G D$ ' is shown as coloured circles in Figure 5, which also shows the Kriging predictions of NS throughput the area, calculated in 'geoR'. The map shows lower NS in the 'south-east' and 'south-west' sites (NS=135-150), with an increase in NS in the 'central' (NS=146-162) and 'north-west' (NS=150-160) sites. The estimated prediction error for ordinary kriging after cross validation indicates that on an average, an error of 2.14 of mean NS can be expected at any given location. Spatial variation in NS was roughly consistent with egg counts (Figure 4), where higher values were observed in the northwest and lower values in the southeast.

NS estimates produced by $S G D$ were lower than using the inverse of the regression slope, which estimated NS = 383 mosquitoes (95\% C.I 287-572) across the study area. 


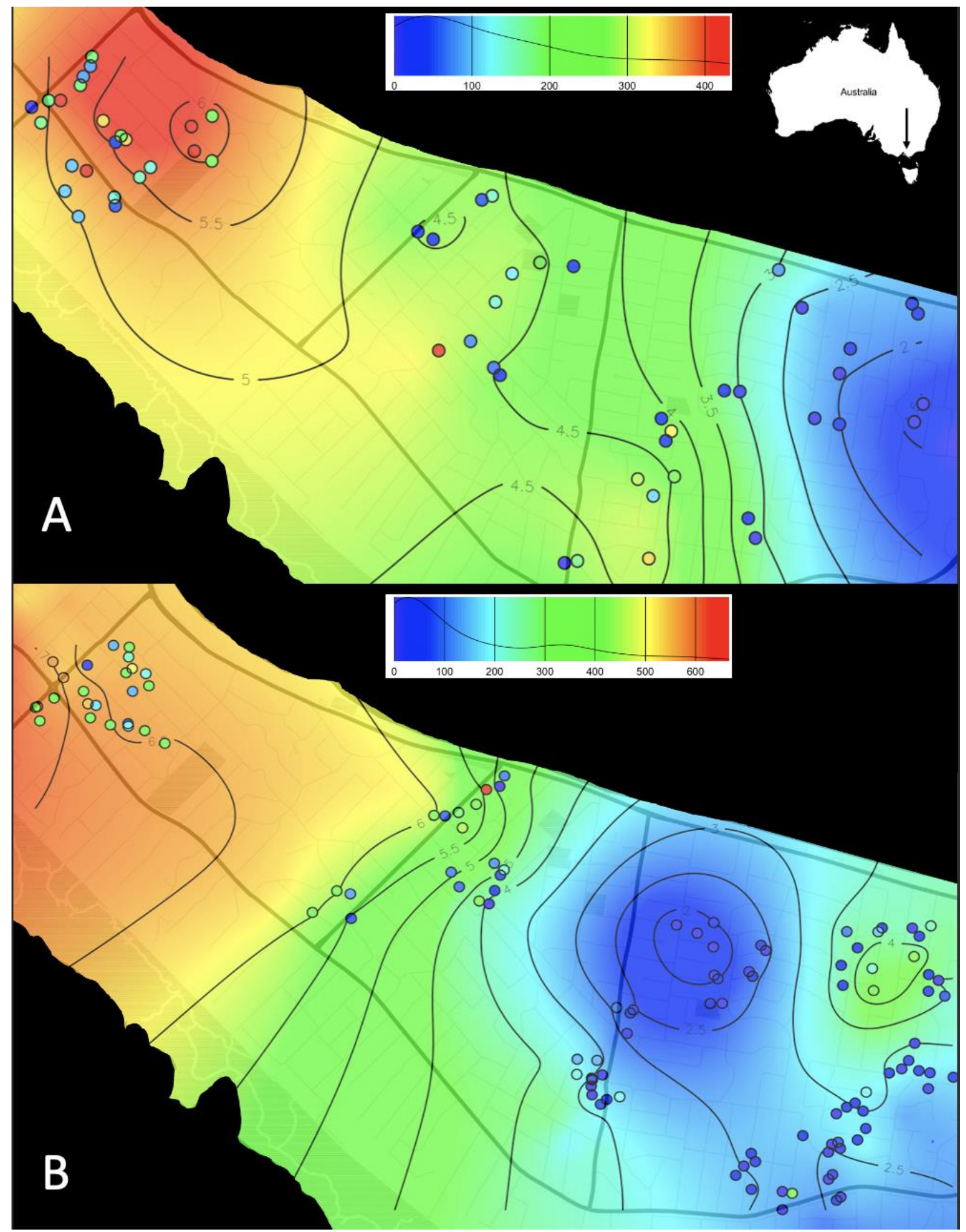

Figure 4: Ordinary Kriging of egg counts throughout the study area at the Mornington Peninsula. Trap

locations are plotted as circles with colours indication the number of eggs per trap. Kriging predictions were performed on cube transformed egg counts and are area shown as blue, green, yellow to red for predicted numbers of eggs from low to high. Distribution of egg counts shown in top-right panels. (A) Eggs collected in November 2019; (B) Eggs collected in February 2020. 


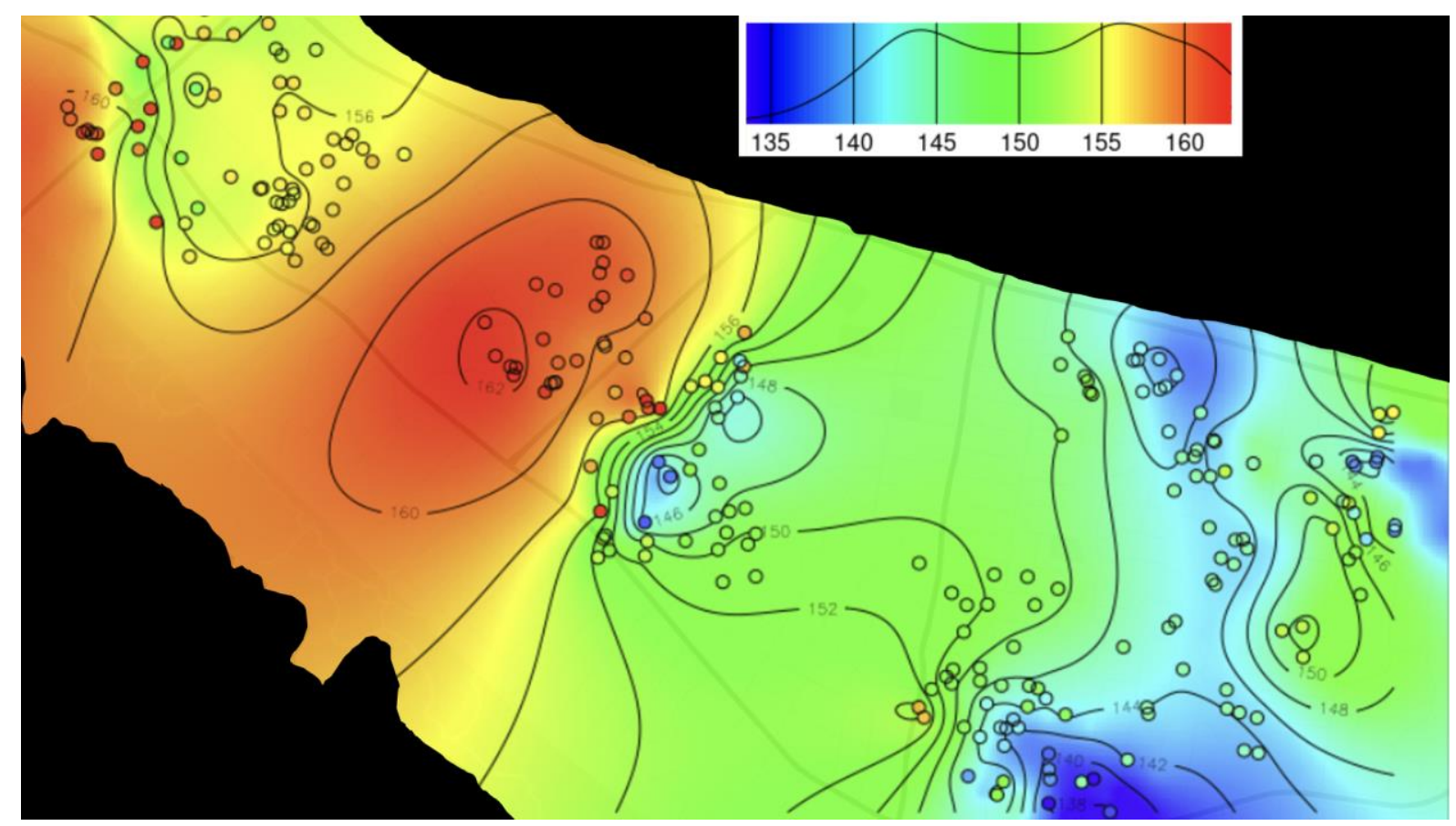

Figure 5: Ordinary Kriging of Neighbourhood size (NS) throughout the study area at the Mornington

Peninsula. Neighbourhood size was calculated for each trap location using $S G D$ in $R$ and are represented as circles. Kriging predictions are shown as blue, green, yellow to red for predicted NS from low to high.

Distribution of NS numbers shown in the top-right panel. NS was calculated from genetic data from individuals collected in February 2019.

\section{Discussion}

The data presented in this study provides important new information about

Ae. notoscriptus, contributing to knowledge of the fundamental ecology of this container breeding mosquito. Our results indicate higher dispersal abilities of Ae. notoscriptus than previously described, which will be important to consider when developing vector control strategies to decrease this species' impact on human and animal health.

Through our kinship analyses, we were able to identify 19 close kin dyads from 240 individual mosquitoes sequenced, including three full-siblings, eight half-siblings and eight first cousins. The spatial separation distance of full-sibling pairs can be interpreted as direct past movement of one individual female mosquito, which moved between two traps in which a full-sibling pair was detected. The maximum distance between full siblings of $1267 \mathrm{~m}$ shows that an individual female was able to disperse between two adjacent sampling sites (Figure $3 \mathrm{~A}$ ) and represents the furthers distance travelled reported for an 
individual Ae. notoscriptus to date. Previous MRR studies reported mean distances travelled by Ae. notoscriptus ranging from $57 \mathrm{~m}$ to $158 \mathrm{~m}$, with $238 \mathrm{~m}$ being the maximum observed distance travelled (Watson et al. 2000c, Trewin et al. 2019). While the mean separation distance of full-siblings calculated in this paper (i.e., $440 \mathrm{~m}$ ) is larger than reported previously by MRR, it is important to note that the 'true' mean separation distance is likely to be smaller than estimated. This is a result of trap placement (i.e., distances between traps) and sample selection (i.e., one individual per trap). However, given that full-sibling pairs were detected at the reported separation distances indicate that Ae. notoscriptus disperses in the described distance ranges commonly, and that individual females are frequently able to move across a sampling site as well as between adjacent sites.

Though distances between half-sibling pairs cannot be directly translated into past individual movement (see 2.2.5: movement of father as well as individual mothers), their separation distances provide an indication about the general movement of Ae. notoscriptus through the study area while still within the same generation. Like full-siblings, we detected most half-sibling pairs within the same sampling site, with some being found between adjacent sites (Figure $3 \mathrm{~A}$ ) which provides additional evidence that movement between adjacent sites occurs commonly. That first cousin pairs were found mostly between sampling sites furthest apart ('north-west' and 'north-east') suggests that it takes approximately two generations for Ae. notoscriptus to disperse through the entire study area. The low $F_{\text {st }}$ values (Table 1) between sampling sites and the lack of clustering in the coancestry analysis provided by FineRADstructure (Figure 1) indicated low genetic structure within sites, which indicates gene flow between sites (Bossart and Prowell 1998) and therefore also points towards high dispersal capacity of Ae. notoscriptus in the study area.

The comparison of spatial autocorrelation between Ae. notoscriptus and Ae. aegypti produced evidence of strong pattern of localized genetic structure in both Ae. aegypti populations, indicating limited dispersal abilities of this species. Because Ae. notoscriptus showed a strong positive Mantel correlation at more than tenfold larger distances than the observed correlation shown in Ae. aegypti, we conclude that Ae. notoscriptus disperses over longer distances than Ae. aegypti (Figure 2). This interpretation is strengthened by the strong IBD detected in both Ae. aegypti datasets, while no IBD could be detected in Ae. 
notoscriptus at similar spatial scales (i.e., within sampling sites). We detected IBD of $A e$. notoscriptus on the scale of the entire dataset (i.e., across all sampling sites) and calculated a neighbourhood size (NS : Wright 1946) of 287-572, which is similar to NS calculated in Ae. aegypti (Jasper et al. 2019) and Ae. albopictus (Schmidt et al. 2021).

While both species have adapted to breed predominantly in artificial containers, there are stark differences in other important ecological factors between Ae. aegypti and Ae. notoscriptus. While Ae. notoscriptus does seeks human hosts for blood feeding, this species reportedly feeds on other animals such as dogs, birds, horses, possums, and fruit bats (Kay et al. 2008), while Aedes aegypti is a highly anthropophilic species (Harrington et al. 2001). This difference in host preferences may contribute to the higher dispersal range of Ae. notoscriptus, as this species is not reliant on human blood meals to breed successfully, hence can move further away from human proximity. Differences in mating behaviour could also contribute to the observed differences in dispersal patterns. While Ae. aegypti is rather easy to adapt to laboratory settings, Watson et al. (2000a) reported that that free mating of colonies was difficult to achieve for Ae. notoscriptus, which could be due to differences in mating behaviour (e.g., male swarming behaviour). Additionally, MRR studies investigating Ae. notoscriptus dispersal reported exceptionally low male recapture rates compared to other Aedes species (Watson et al. 2000b, Trewin et al. 2019), which could mean that male Ae. notoscriptus respond to different cues than other Aedes mosquitoes.

The high mobility of Ae. notoscriptus at the Mornington Peninsula could have influenced the success of the mosquito control pilot trial, conducted in early 2021. When comparing the size of the intervention zones ( $250 \mathrm{~m} \times 350 \mathrm{~m})$ with the spatial autocorrelation of genetic distances (Figure 2) and the separation distances of kin dyads estimated in this study (Figure 3), the scale of movement of Ae. notoscriptus appears to exceed the size of control zones. As a result, zones were likely to be invaded by mosquitoes from surrounding areas while control measures were in place, which could have compromised the success of lowering mosquito number in controlled zones. Controlled zones were also likely to be re-invaded within a month or two after the trial, given that the generation time of Ae. notoscriptus of approximately one month. The control of Ae. notoscriptus on the block level has already been questioned by Trewin et al. (2019) who argue that while Ae. aegypti may be controlled 
in that way, the control of a highly dispersive container breeder such as Ae. notoscriptus will likely require much bigger areas, which is expected to be expensive and labour intensive. Moving forward, alternative approaches should be considered to control Ae. notoscriptus, such as Wolbachia mediated manipulation of populations, or the strategic use of chemical control measures.

To investigate whether number of eggs can be used to infer relative population densities of Ae. notoscriptus, we used ordinary Kriging predictions on egg count data from two individual sampling efforts and compared their spatial pattern to predictions calculated from spatially explicit estimations of NS, calculated by 'sGD'. We found a consistent trend of lower predicted egg numbers in the 'north-east' and 'south-east' sampling sites with increasing number of eggs through the 'central' to the 'north-west' sites at both timepoints (i.e., November 2019 and February 2020) (Figure 4 A+B). The pattern of our NS predictions, inferred from the molecular data from collections in February 2019 follow this trend, indicating lower numbers of mosquitoes in the 'north-east' and 'south-east', compared to the 'central' and 'north-west' sites (Figure 5). While this comparison provides an indication that higher egg counts correlate with a larger NS, the results need to be interpreted with caution as egg counts and molecular data were acquired in separate sampling efforts and therefore, do not represent a direct comparison. Previous studies presented a positive relationship between Aedes egg numbers collected by oviposition traps and numbers of adult mosquitoes collected in adult traps (Tantowijoyo et al. 2016, Feria-Arroyo et al. 2020). Garcia et al. (2020) reported a mismatch of egg counts between oviposition traps and adult mosquitoes collected, however the use of direct aspiration for two hours on a single day compared to oviposition traps that were in place for five consecutive days may have influenced the comparability of their results. The comparison of NS through continuous populations inferred from molecular data to numbers of eggs collected in oviposition traps presents a great opportunity to gather more evidence for the link between the number of mosquitoes and eggs counts and should be considered in future.

We presented evidence of high dispersal abilities of Ae. notoscriptus at the Mornington peninsula and highlighted the importance of acquiring species specific ecological information when planning management strategies. We showed that generalisations, even 
between related species, can be misleading and should be interpreted with caution if used to plan interventions. Finally, we provide evidence that egg counts collected by oviposition traps can be linked to NS and could therefore be used to estimate the impact of control measures.

\section{Acknowledgments}

We thank Nancy Endersby-Harshman and Qiong Yang for laboratory assistance and Moshe Jasper for helpful discussions. This research was funded by a University of Melbourne Early Career Researcher grant awarded to TLS. VP was financially supported by the Australian Government Research Training Program Scholarship.

\section{References}

1. Beier, J. C. 2003. Malaria parasite development in mosqutioes. http://dx.doi.org/10.1146/annurev.ento.43.1.519 43:519-543.

2. Bhatt, S., P. W. Gething, O. J. Brady, J. P. Messina, A. W. Farlow, C. L. Moyes, J. M. Drake, J. S. Brownstein, A. G. Hoen, O. Sankoh, M. F. Myers, D. B. George, T. Jaenisch, G. R. William Wint, C. P. Simmons, T. W. Scott, J. J. Farrar, and S. I. Hay. 2013. The global distribution and burden of dengue. Nature 496:504.

3. Bossart, J. L., and D. Pashley Prowell. 1998. Genetic estimates of population structure and gene flow: Limitations, lessons and new directions. Trends in Ecology \& Evolution 13:202206.

4. Bravington, M. V., H. J. Skaug, and E. C. Anderson. 2016. Close-Kin Mark-Recapture. https://doi.org/10.1214/16-STS552 31:259-274.

5. Browning, B. L., and S. R. Browning. 2016. Genotype Imputation with Millions of Reference Samples. The American Journal of Human Genetics 98:116-126.

6. Catchen, J., P. A. Hohenlohe, S. Bassham, A. Amores, and W. A. Cresko. 2013. Stacks: an analysis tool set for population genomics. Molecular Ecology 22:3124-3140.

7. Combs, M., E. E. Puckett, J. Richardson, D. Mims, and J. Munshi-South. 2018. Spatial population genomics of the brown rat (Rattus norvegicus) in New York City. Molecular Ecology 27:83-98.

8. Conomos, M. P., A. P. Reiner, B. S. Weir, and T. A. Thornton. 2016. Model-free Estimation of Recent Genetic Relatedness. The American Journal of Human Genetics 98:127-148. 
9. Christophers, S. R. 1960. Aëdes aegypti (L.) the Yellow Fever Mosquito: its Life History, Bionomics and Structure. Cambridge University Press.

10. Danecek, P., J. K. Bonfield, J. Liddle, J. Marshall, V. Ohan, M. O. Pollard, A. Whitwham, T. Keane, S. A. McCarthy, R. M. Davies, and H. Li. 2021. Twelve years of SAMtools and BCFtools. GigaScience 10:1-4.

11. Doak, D. F., P. C. Marino, and P. M. Kareiva. 1992. Spatial scale mediates the influence of habitat fragmentation on dispersal success: Implications for conservation. Theoretical Population Biology 41:315-336.

12. Dobrotworsky, N. V. 1965. The mosquitoes of Victoria (Diptera, Culicidae). The mosquitoes of Victoria (Diptera, Culicidae).

13. Doggett, S. L., and R. C. Russell. 1997. Aedes notoscriptus can transmit inland and coastal isolates of Ross River and Barmah Forest viruses from New South Wales. Arbovirus Res Aust 7:79-81.

14. Dray, S., and A.-B. Dufour. 2007. Journal of Statistical Software The ade4 Package: Implementing the Duality Diagram for Ecologists.

15. Edland, T. 1983. Attacks by the winter moth group (Operophtera brumata, Agriopis aurantiaria, Erannis defoliaria) in orchards. A system for forecasting the expected attack degree. Gartneryrket 73:208-212.

16. Feria-Arroyo, T., C. Aguilar, C. Q. Vazquez, R. Santos-Luna, S. Roman-Perez, T. Oraby, G. S. Tejeda, F. C. Morales, V. S. Bueyes, and P. C. Guevara. 2020. A tale of two cities: Aedes Mosquito surveillance across the Texas-Mexico Border. Subtropical Agriculture and Environments 71.

17. Fountain, T., A. Husby, E. Nonaka, M. F. DiLeo, J. H. Korhonen, P. Rastas, T. Schulz, M. Saastamoinen, and I. Hanski. 2018. Inferring dispersal across a fragmented landscape using reconstructed families in the Glanville fritillary butterfly. Evolutionary Applications 11:287297.

18. Garcia, K. K. S., H. S. Versiani, T. O. Araújo, J. P. A. Conceição, M. T. Obara, W. M. Ramalho, T. T. C. Minuzzi-Souza, G. D. Gomes, E. N. Vianna, R. V. Timbó, V. G. C. Barbosa, M. S. P. Rezende, L. P. F. Martins, G. O. Macedo, B. L. Carvalho, I. M. Moreira, L. A. Bartasson, N. Nitz, S. L. B. Luz, R. Gurgel-Gonçalves, and F. Abad-Franch. 2020. Measuring mosquito control: Adult-mosquito catches vs egg-trap data as endpoints of a cluster-randomized controlled trial of mosquito-disseminated pyriproxyfen. Parasites and Vectors 13:1-15.

19. Goldberg, E. E., and R. Lande. 2015. Species' Borders and Dispersal Barriers. https://doi.org/10.1086/518946 170:297-304. 
20. Goslee, S. C., and D. L. Urban. 2007. The ecodist Package for Dissimilarity-based Analysis of Ecological Data. Journal of Statistical Software 22:1-19.

21. Hardy, O. J., and X. Vekemans. 2002. spagedi: a versatile computer program to analyse spatial genetic structure at the individual or population levels. Molecular Ecology Notes 2:618-620.

22. Harrington, L. C., J. D. Edman, and T. W. Scott. 2001. Why Do Female Aedes aegypti (Diptera: Culicidae) Feed Preferentially and Frequently on Human Blood? Journal of Medical Entomology 38:411-422.

23. Hoffmann, A. A., B. L. Montgomery, J. Popovici, I. Iturbe-Ormaetxe, P. H. Johnson, F. Muzzi, M. Greenfield, M. Durkan, Y. S. Leong, Y. Dong, H. Cook, J. Axford, A. G. Callahan, N. Kenny, C. Omodei, E. A. McGraw, P. A. Ryan, S. A. Ritchie, M. Turelli, and S. L. O’Neill. 2011. Successful establishment of Wolbachia in Aedes populations to suppress dengue transmission. Nature 2011 476:7361 476:454-457.

24. Jasper, M., T. L. Schmidt, N. W. Ahmad, S. P. Sinkins, and A. A. Hoff-. 2019. A genomic approach to inferring kinship reveals limited intergenerational dispersal in the yellow fever mosquito.

25. Jeger, M. J. 1999. Improved understanding of dispersal in crop pest and disease management: current status and future directions. Agricultural and Forest Meteorology 97:331-349.

26. Johnson, M. T. J., and J. Munshi-South. 2017. Evolution of life in urban environments. Science 358.

27. Juarez, J. G., L. F. Chaves, S. M. Garcia-Luna, E. Martin, I. Badillo-Vargas, M. C. I. Medeiros, and G. L. Hamer. 2021. Variable coverage in an Autocidal Gravid Ovitrap intervention impacts efficacy of Aedes aegypti control. Journal of Applied Ecology 58:2075-2086.

28. Kay, B. H., T. M. Watson, and P. A. Ryan. 2008. Definition of productive Aedes notoscriptus (Diptera: Culicidae) habitats in western Brisbane, and a strategy for their control. Australian Journal of Entomology 47:142-148.

29. Killeen, G. F., B. G. J. Knols, and W. Gu. 2003. Taking malaria transmission out of the bottle: implications of mosquito dispersal for vector-control interventions. The Lancet Infectious Diseases 3:297-303.

30. Langmead, B., and S. L. Salzberg. 2012. Fast gapped-read alignment with Bowtie 2. Nature methods 9:357.

31. Lee, D. J., M. M. Hicks, M. Griffiths, M. L. Debenham, J. H. Bryan, R. C. Russel, and M. Geary. 1987. The Culicidae of the Australasian Region: Genus Anopheles (Anopheles, Cellia). Page 
Monograph Series, Entomology Monograph. Australian Government Publishing Service.

32. Malinsky, M., E. Trucchi, D. J. Lawson, and D. Falush. 2018. RADpainter and

fineRADstructure: Population Inference from RADseq Data. Molecular Biology and Evolution 35:1284-1290.

33. McCarroll, L., M. G. Paton, S. H. P. P. Karunaratne, H. T. R. Jayasuryia, K. S. P. Kalpage, and J. Hemingway. 2000. Insecticides and mosquito-borne disease. Nature 2000 407:6807 407:961-962.

34. Metzger, M. E., J. W. Wekesa, S. Kluh, K. K. Fujioka, R. Saviskas, A. Arugay, N. McConnell, K. Nguyen, L. Krueger, G. M. Hacker, R. Hu, and V. L. Kramer. 2021. Detection and Establishment of Aedes notoscriptus (Diptera: Culicidae) Mosquitoes in Southern California, United States. Journal of Medical Entomology.

35. Muir, L. E., and B. H. Kay. 1998. Aedes aegypti survival and dispersal estimated by markrelease-recapture in northern Australia. American Journal of Tropical Medicine and Hygiene $58: 277-282$.

36. Ritchie, S. A. 2001. Scientific note effect of some ainimal feeds and oviposition subrtrates on Aedes oviposition in ovitraps in Cairns, Australia. Journal of the American Mosquito Contol Association 11:2.

37. Rousset. 2000. Genetic differentiation between individuals. J Evol Biol 13:58-62.

38. Russell, R. C., and Geary M. J. The susceptibility of the mosquitoes Aedes notoscriptus and Culex annulirostris to infection with dog heartworm Dirofilaria immitis and their vector efficiency. Medical and Veterinary Entomology 6:154-158.

39. Saddler, A., K. S. Kreppel, N. Chitnis, T. A. Smith, A. Denz, J. D. Moore, M. M. Tambwe, and S. J. Moore. 2019. The development and evaluation of a self-marking unit to estimate malaria vector survival and dispersal distance. Malaria Journal 18:1-14.

40. Schmidt, T. L., I. Filipović, A. A. Hoffmann, and G. Rašić. 2018a. Fine-scale landscape genomics helps explain the slow spatial spread of Wolbachia through the Aedes aegypti population in Cairns, Australia. Heredity 120:386-395.

41. Schmidt, T. L., I. Filipović, A. A. Hoffmann, and G. Rašić. 2018b. Fine-scale landscape genomics helps explain the slow spatial spread of Wolbachia through the Aedes aegypti population in Cairns, Australia. Heredity 2018 120:5 120:386-395.

42. Schmidt, T. L., T. Swan, J. Chung, S. Karl, S. Demok, Q. Yang, M. A. Field, M. O. Muzari, G. Ehlers, M. Brugh, R. Bellwood, P. Horne, T. R. Burkot, S. Ritchie, and A. A. Hoffmann. 2021. Spatial population genomics of a recent mosquito invasion. Molecular Ecology 30:11741189. 
43. Shirk, A. J., and S. A. Cushman. 2011. sGD: software for estimating spatially explicit indices of genetic diversity. Molecular Ecology Resources 11:922-934.

44. Shirk, A. J., and S. A. Cushman. 2014. Spatially-explicit estimation of Wright's neighborhood size in continuous populations. Frontiers in Ecology and Evolution 2:62.

45. Sunahara, T., and M. Mogi. 2004. Searching clusters of community composition along multiple spatial scales: a case study on aquatic invertebrate communities in bamboo stumps in West Timor. Population Ecology 2004 46:2 46:149-158.

46. T.M., W., and K. B.H. 1999. Vector competence of Aedes notoscriptus (Diptera: Culicidae) for Barmah Forest virus and of this species and Aedes aegypti (Diptera: Culicidae) for dengue 14 viruses in Queensland, Australia. Journal of medical entomology 36:508-514.

47. Tantowijoyo, W., E. Arguni, P. Johnson, N. Budiwati, P. I. Nurhayati, I. Fitriana, S. Wardana, H. Ardiansyah, A. P. Turley, P. Ryan, S. L. O’Neill, and A. A. Hoffmann. 2016. Spatial and Temporal Variation in Aedes aegypti and Aedes albopictus (Diptera: Culicidae) Numbers in the Yogyakarta Area of Java, Indonesia, With Implications for Wolbachia Releases. Journal of Medical Entomology 53:188-198.

48. Thomas, C. J., D. E. Cross, and C. Bøgh. 2013. Landscape Movements of Anopheles gambiae Malaria Vector Mosquitoes in Rural Gambia. PLOS ONE 8:e68679.

49. Trense, D., T. L. Schmidt, Q. Yang, J. Chung, A. A. Hoffmann, and K. Fischer. 2021. Anthropogenic and natural barriers affect genetic connectivity in an Alpine butterfly. Molecular Ecology 30:114-130.

50. Trewin, B., D. E. Pagendam, J. M. Darbro, Q. Health, and G. J. Devine. 2019. Urban Landscape Features Influence the Movement and Distribution of the Australian Container-Inhabiting Mosquito Vectors Aedes aegypti (Diptera: Culicidae) and Aedes notoscriptus ( Epidemiology of Ross River virus in South East Queensland, Australia View project Non-human reservoirs of zoonotic pathogens View project. Article in Journal of Medical Entomology.

51. Verdonschot, P. F. M., and A. A. Besse-Lototskaya. 2014. Flight distance of mosquitoes (Culicidae): A metadata analysis to support the management of barrier zones around rewetted and newly constructed wetlands. Limnologica 45:69-79.

52. Watson, T. M., K. L. Marshall, and B. H. Kay. 2000a. Colonization and laboryatory biology of Aedes notoscriptus from Brisbane, Australia. Journal of the American Mosquito Control Association 16:138-142.

53. Watson, T. M., A. Saul, and B. H. Kay. 2000b. Aedes notoscriptus (Diptera: Culicidae) Survival and Dispersal Estimated by Mark-Release-Recapture in Brisbane, Queensland, Australia. J. Med. Entomol 37:380-384. 
bioRxiv preprint doi: https://doi.org/10.1101/2022.01.18.476837; this version posted January 21, 2022. The copyright holder for this preprint (which was not certified by peer review) is the author/funder, who has granted bioRxiv a license to display the preprint in perpetuity. It is made available under aCC-BY-NC 4.0 International license.

54. Webb, C. E., S. L. Doggett, and R. C. Russell. (n.d.). A guide to the mosquitoes of Australia.

55. Wright, S. 1946. Isolation by Distance under Diverse Systems of Mating. Genetics 31:39. 